\title{
Dessecação e viabilidade de sementes de gravioleira
}

\author{
Desiccation and viability of graviola seeds \\ G. R. G. Figueiredo ${ }^{1}$; C. E. Pereira ${ }^{2 *}$; C. K. Sacramento ${ }^{1}$ \\ ${ }^{1}$ Universidade Estadual de Santa Cruz, Departamento de Ciências Agrárias e Ambientais \\ ${ }^{2}$ Universidade Federal do Sul da Bahia, Centro de Formação em Ciências Agroflorestais \\ *ce-pereira@bol.com.br
}

(Recebido em 25 de setembro de 2018; aceito em 27 de junho de 2019)

\begin{abstract}
Objetivou-se avaliar a tolerância à dessecação e a viabilidade de sementes de gravioleira em diferentes substratos. Inicialmente determinou-se o teor de água das sementes e, em seguida, foram submetidas à secagem até atingirem o teor de água de 31\%, 25,1\%, 19,3\%, 15,5\%, 9,5\% e 7\%. Como substratos foram utilizados areia e substrato comercial. As sementes foram analisadas por meio da emergência, índice de velocidade de emergência e do comprimento e massa de matéria seca de plântulas. As sementes de graviola são tolerantes à desidratação até pelo menos $7 \%$ de teor de água. $\mathrm{O}$ melhor substrato para germinação foi o comercial para todas as variáveis avaliadas.

Palavras-chave: Annona muricata L., graviola, secagem
\end{abstract}

It was evaluated the effects of dehydration and the viability of graviola seeds, placed on different substrates. It was first determined the water content of these seeds were then subjected to drying equipment with forced air circulation until they reach the water content of $31 \%, 25.1 \%, 19.3 \%, 15.5 \%, 9.5 \%$ and $7 \%$ and placed to emerge in sand and commercial substrate. The seeds were evaluated by emergence, emergence rate index, length and dry mass of seedlings. Graviola seeds are tolerant to dehydration up to at least $7 \%$ water content. The best substrate for germination was commercial for all evaluated variables.

Keywords: Annona muricata L., graviola, drying

\section{INTRODUÇÃO}

Para as espécies tropicais nativas, o conhecimento do menor teor de água suportável pelas sementes, sem que haja o comprometimento da qualidade fisiológica é imprescindível para definir a melhor tecnologia de armazenamento $[1,2]$.

Nas fases iniciais de desenvolvimento das sementes as mesmas não possuem completa tolerância à dessecação [3], e com a maturação, para a maioria das espécies, as sementes adquirem tolerância à dessecação a teores de água próximos de $2 \%$ a 5\%, ou mesmo abaixo desses níveis, sendo denominadas ortodoxas. Outras espécies toleram dessecação a teores de água em torno de $10 \%$ a $13 \%$, tendo a viabilidade reduzida em níveis inferiores, classificadas como intermediárias, e sementes que não toleram dessecação a abaixo de $20 \%$ e armazenamento a baixas temperaturas, denominadas recalcitrantes [4,5]. Algumas espécies dentro da família da Annonaceae apresentam sementes ortodoxas, dentre elas a Annona glabra L. [6], Xylopia aethiopica (Dunal) A Rich [7] e Annona emarginata (Schltdl.) H. Rainer [8].

De acordo com as orientações contidas nas Regras para Análise de Sementes [9] além da luz, temperatura, água e oxigênio, a escolha do substrato tem fundamental importância nos resultados de germinação. $O$ tipo de substrato utilizado para a germinação das sementes afeta o desempenho das mesmas, neste sentido, autores verificaram que o substrato comercial proporcionou melhor desempenho das sementes e plantas de pinheira (Annona squamosa L.), sendo recomendado para a produção de mudas desta espécie [10].

Assim, o objetivo deste trabalho foi estudar os efeitos da desidratação sob diferentes níveis de teor de água sobre o comportamento fisiológico das sementes de gravioleira em areia e substrato comercial. 


\section{MATERIAL E MÉTODOS}

Os frutos utilizados no experimento foram colhidos em estado maduro, em gravioleiras do tipo 'Morada', cultivadas em Ubatã, Bahia. Após a coleta, os frutos foram transportados para o laboratório, onde foi efetuada manualmente a extração, lavagem e seleção, eliminando-se as sementes malformadas ou parcialmente danificadas. Em seguida as sementes foram secas superficialmente com papel absorvente por cerca de 10 minutos.

Do lote de sementes foram retiradas quatro amostras de $15 \mathrm{~g}$ e determinado o teor de água inicial [9]. As sementes restantes foram distribuídas formando uma única camada, sem sobreposição das sementes, sobre bandejas de alumínio e os tratamentos foram obtidos por meio do acompanhamento da perda de água das sementes durante a secagem em estufa de circulação forçada de ar a $32 \pm 2^{\circ} \mathrm{C}$. Para monitorar o processo, amostras de sementes com massa inicial conhecida foram colocadas em placas de Petri e distribuídas nas prateleiras da estufa para pesagem, com auxílio de balança analítica. A massa final das amostras, correspondente aos teores de água desejados, foi previamente determinada.

Porém cada tratamento foi definido após a determinação do teor de água das sementes pelo método padrão da estufa a $105 \pm 3^{\circ} \mathrm{C}$ por 24 horas [9], utilizando-se quatro repetições com $15 \mathrm{~g}$ de sementes cada. Os resultados, expressos em porcentagem, foram calculados com base na massa úmida $(\mathrm{Bu})$, por meio da equação:

$$
\text { T.A. }=100(\mathrm{Pi}-\mathrm{Pf}) /(\mathrm{T}-\mathrm{Pi})
$$

onde: T.A. $=$ Teor de água $(\%) ; \mathrm{Pi}=$ Peso inicial; $\mathrm{Pf}=$ Peso final $\mathrm{e} \mathrm{T}=$ Peso do recipiente.

Assim, buscou-se obter teores de água das sementes em intervalos regulares a partir do teor obtido logo após a colheita e beneficiamento e, com isso foram utilizados os seguintes teores de água: $31,0 \% ; 25,1 \% ; 19,3 \% ; 15,5 \% ; 9,5 \%$ e $7,0 \%$.

Amostras de sementes com cada teor de água obtido foram semeadas em bandejas de células de poliestireno, devidamente identificadas, contendo areia ou substrato comercial e mantidas em bancadas na casa de vegetação. Foi utilizada areia de textura média, peneirada em malha de 0,8

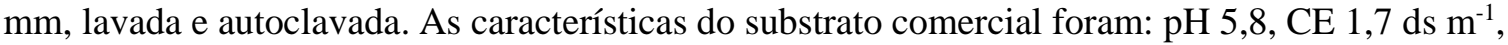
densidade $500 \mathrm{~kg} \mathrm{~m}^{-3}$. A capacidade de retenção de água (retenção a $0,01 \mathrm{MPa}$ ) foi determinada para areia e substrato comercial segundo EMBRAPA (2011) [11]. Ambos os substratos foram umedecidos diariamente com auxílio de regador contendo água destilada até a saturação. A semeadura foi realizada a $1 \mathrm{~cm}$ de profundidade.

Para avaliação dos tratamentos, foram realizadas as avaliações de emergência de plântulas: as avaliações foram realizadas aos 85 dias após a semeadura (DAS), e o critério utilizado foi o de plântulas normais emersas [9], e os resultados, expressos em porcentagem.

Índice de velocidade de emergência de plântulas (IVE): calculado de acordo com a fórmula proposta por Maguire (1962) [12], foi baseado na leitura diária do número de sementes com epicótilo emergido, a partir do $15^{\circ}$ até o $85^{\circ}$ dia após a semeadura (DAS).

Comprimento de plântulas: realizado aos $85 \mathrm{DAS}$, onde o comprimento da raiz até a inserção da última folha das plântulas normais foi medido com o auxílio de uma régua graduada em centímetros.

Massa de matéria seca de plântulas: as plântulas normais coletadas aos 85 DAS foram colocadas em sacos de papel e levadas à estufa com circulação forçada de ar, à $65^{\circ} \mathrm{C}$, permanecendo até atingir peso constante, quando foram pesadas em balança semi-analítica. Os resultados foram expressos em gramas.

Foi utilizado esquema fatorial $6 \times 2$ em delineamento inteiramente casualizado, sendo, 6 teores de água das sementes $(31,0 \% ; 25,1 \% ; 19,3 \% ; 15,5 \% ; 9,5 \%$ e $7,0 \%)$ e 2 substratos (areia e substrato comercial), totalizando 12 tratamentos, o quais foram avaliados utilizando-se quatro repetições com 25 sementes por parcela.

Os dados foram submetidos à análise de variância e as médias comparadas pelos testes de $\mathrm{F} \mathrm{e}$ Tukey, a 5\% de probabilidade. As análises foram realizadas utilizando-se o programa computacional SISVAR [13]. 


\section{RESULTADOS E DISCUSSÃO}

Pela análise de variância, observou-se efeito significativo da interação teor de água e substratos apenas para o índice de velocidade de emergência (IVE), enquanto foi observada significância para a fonte de variação substrato para todos os parâmetros avaliados e apenas no IVE para o teor de água.

Independente do teor de água e do substrato, a emergência das sementes de gravioleira iniciou aos 15 dias após a semeadura, estabilizando aos 75 dias no substrato comercial. Esses resultados estão de acordo com Sacramento et al. (2009) [14] que, trabalhando com sementes da mesma espécie, relatam início da emergência entre 10 e 20 dias, dependendo da temperatura do ambiente. Também Oliveira et al. (2005) [15] verificaram para sementes de Annona montana Macf. que a germinação iniciou cerca de 28 dias após a semeadura, sendo que a temperatura de $30^{\circ} \mathrm{C}$ possibilitou a maior porcentagem de germinação.

Pôde-se observar que as sementes colocadas em substrato comercial, independente do teor de água das mesmas, tiveram significativamente maior porcentagem de emergência $(94,3 \pm 1,99)$ quando comparadas às sementes semeadas em areia (Tabela 1).

Tabela 1. Resultados da porcentagem de emergência, comprimento de plântulas e massa seca de plântulas oriundas de sementes de gravioleira (Annona muricata L.) com diferentes teores de água e submetidas a dois substratos para germinação.

\begin{tabular}{lccc}
\hline Substratos & $\begin{array}{c}\text { Emergência } \\
(\boldsymbol{\%})\end{array}$ & $\begin{array}{c}\text { Comprimento de plântulas } \\
(\mathbf{c m})\end{array}$ & $\begin{array}{c}\text { Massa seca de plântula } \\
(\mathbf{g})\end{array}$ \\
\hline Areia & $80,2 \mathrm{~b}$ & $18,13 \mathrm{~b}$ & $4,76 \mathrm{~b}$ \\
Substrato comercial & $94,3 \mathrm{a}$ & $20,54 \mathrm{a}$ & $7,21 \mathrm{a}$ \\
\hline Teor de água (\%) & & & \\
\hline 31,0 & $91,5 \mathrm{a}$ & $19,58 \mathrm{a}$ & $6,43 \mathrm{a}$ \\
25,5 & $85,0 \mathrm{a}$ & $17,57 \mathrm{a}$ & $5,98 \mathrm{a}$ \\
19,3 & $81,5 \mathrm{a}$ & $18,86 \mathrm{a}$ & $5,56 \mathrm{a}$ \\
15,5 & $90,0 \mathrm{a}$ & $20,24 \mathrm{a}$ & $6,41 \mathrm{a}$ \\
9,5 & $85,0 \mathrm{a}$ & $19,80 \mathrm{a}$ & $5,91 \mathrm{a}$ \\
7,0 & $90,5 \mathrm{a}$ & $19,95 \mathrm{a}$ & $5,64 \mathrm{a}$ \\
\hline $\mathrm{CV}(\%)$ & 11,2 & 9,6 & 13,6 \\
\hline
\end{tabular}

Médias seguidas pela mesma letra minúscula para os fatores substratos e teores de água, não diferem significativamente entre si ao nível de 5\% de probabilidade pelo teste de F e de Tukey, respectivamente.

O substrato comercial foi superior à areia para as variáveis comprimento de plântula e massa seca de plântula. Durante o experimento observou-se a que o substrato comercial apresentou uma maior retenção máxima de água, de $52 \%$ e $30 \%$ no intervalo de 24 horas, enquanto a areia apresentou retenção máxima de $22 \%$ e $7 \%$ no mesmo período. Com relação à temperatura o substrato apresentou $1,5^{\circ} \mathrm{C}$ acima da temperatura da areia, considerando que a temperatura no substrato era $1^{\circ} \mathrm{C}$ abaixo da temperatura do ar. Andrade et al. (2000) [16] afirmam que substratos comerciais são constituídos por partículas maiores, apresentam maior espaço vazio, menor densidade aparente (ou menor grau de compactação), maior arejamento e, portanto, maiores facilidades para a emergência das plântulas. As fases iniciais de germinação e emergência não são afetadas pela disponibilidade de nutrientes no substrato, já que os tecidos de reserva das sementes são capazes de suprir tais demandas. Entretanto, com o passar do tempo as plântulas começam a absorver e utilizar nutrientes do meio externo, de modo que características relacionadas ao crescimento das plantas, tais como o comprimento e massa seca de plântulas, podem ser favorecidas, significativamente pela disponibilidade de nutrientes no substrato comercial.

Não houve efeito da dessecação sobre a porcentagem de emergência das sementes de gravioleira, assim pode-se inferir que as mesmas têm características ortodoxas. Durante o desenvolvimento das sementes, ocorre o acúmulo de matéria seca e, em contrapartida a perda de água [17]. Para as sementes de gravioleira, verificou-se tolerância à dessecação até pelo menos $7 \%$ de teor de água. 
Pelos resultados do índice de velocidade de emergência verificou-se que o substrato comercial teve significativamente maiores médias em relação ao substrato areia, independentemente do teor de água das sementes (Tabela 2). Com relação ao teor de água, não houve diferença entre os mesmos quando as sementes foram semeadas em areia, enquanto as sementes com teor de água de 25,0\% tiveram maior índice de velocidade de emergência em relação às demais quando foram submetidas ao substrato comercial.

Tabela 2. Resultado do índice de velocidade de emergência em sementes de gravioleira (Annona muricata L.) com diferentes teores de umidade e substratos de semeadura.

\begin{tabular}{lcccccc}
\hline Substrato & \multicolumn{7}{c}{ Teor de água (\%) } \\
\cline { 2 - 7 } & 31,0 & 25,5 & 19,3 & 15,5 & 9,5 & 7,0 \\
\hline Areia & $0,694 \mathrm{Ab}$ & $0,619 \mathrm{Ab}$ & $0,527 \mathrm{Ab}$ & $0,643 \mathrm{Ab}$ & $0,550 \mathrm{Ab}$ & $0,461 \mathrm{Ab}$ \\
Comercial & $1,304 \mathrm{Ba}$ & $2,791 \mathrm{Aa}$ & $1,138 \mathrm{BCa}$ & $1,058 \mathrm{BCa}$ & $1,098 \mathrm{BCa}$ & $0,894 \mathrm{Ca}$ \\
\hline CV $(\%)$ & \multicolumn{7}{c}{13,7} \\
\hline
\end{tabular}

Médias seguidas pela mesma letra maiúscula na linha e minúscula na coluna, não diferem significativamente entre si, ao nível de $5 \%$ de probabilidade, pelos testes de Tukey e de F, respectivamente.

Braga Júnior et al. (2010) [18] verificaram que não houve diferença entre os resultados na emergência de plântulas de Zizyphus joazeiro Mart., quando semeadas em areia e substrato comercial. O mesmo foi observado em estudo do vigor e emergência de sementes de mulungu (Erythrina velutina Willd), porém para outras características avaliadas como, altura de planta e massa seca total, o substrato comercial foi superior a areia [19].

Resultados semelhantes foram observados quando avaliando o desempenho de sementes de sapota preta (Diospyros ebenaster Retz) em diferentes substratos, onde se observou que os melhores resultados foram na utilização do substrato comercial, superando fibra de coco; areia; substrato mistura de solo com areia e esterco [20].

\section{CONCLUSÃO}

Os teores de água da semente de gravioleira não afetam sua qualidade fisiológica entre os limites de $7 \%$ a $31 \%$, logo após a secagem e o substrato comercial possibilita significativamente maior emergência e crescimento das plântulas de gravioleira.

\section{REFERÊNCIAS BIBLIOGRÁFICAS}

1. Nascimento WMO, Novembre ADL, Cicero SM. Consequências fisiológicas da dessecação em sementes de açaí (Euterpe oleracea Mart.). Rev Bras Sementes. 2007 Ago;29(2):38-43, doi: 10.1590/S010131222007000200006

2. Barbedo CJ, Centeno DC, Ribeiro RCLF. Do recalcitrant seeds really exist?. Hoehnea. Dec;2013 40(4):583-593, doi: 10.1590/S2236-89062013000400001.

3. Gruwez R, Leroux O, Frenne P, Tack W, Viane R, Verheyen K. Critical phases in the seed development of common juniper (Juniperus communis). Plant Biol. 2013 Jun;15(1):210-219. doi: 10.1111/j.14388677.2012.00628.x.

4. Ellis RH, Hong TD, Roberts H. An intermediate category of seed storage behaviour: I. Coffee. J Experim Bot .1990 Sep;41(230):1167-1174. doi: 10.1093/jxb/41.9.1167.

5. Hong TD, Ellis RH. A protocol to determine seed storage behaviour. Rome: International Plant Genetic Resources Institute, 1996. 55p.

6. Carvalho JEU, Nascimento WMO, Muller CH. Tolerância de sementes de araticum-do-brejo (Annona glabra L.) ao dessecamento e ao congelamento. Rev Bras Frutic. 2001 Abr;23(1):179-182.

7. Kanmegne G, Fotso DAM, Omokolo DN. Effects of substrates, different pretreatment protocols and dehydration on the induction of seeds germination of Xylopia aethiopica (Dunal) A. Rich. International J Biol Chem Scien. 2017 11(2):597-608, doi: 10.4314/ijbcs.v11i2.6.

8. Corsato JM. Tolerância à dessecação e armazenamento de sementes de araticum-de-terra-fria (Annona emarginata (SCHLTDL.) H. Rainer) [dissertação]. Botucatu (SP): Universidade Estadual Paulista; 2010. $95 \mathrm{p}$. 
9. Brasil. Regras para análise de sementes. Ministério da Agricultura, Pecuária e Abastecimento - Secretaria de Defesa Agropecuária, Brasília, Brasil. 2009. 399 p.

10. Wagner Júnior A, Neres CRL, Negreiros JRS, Alexandre RS, Diniz ER, Pimentel LD, Bruckner CH. Substratos na formação de mudas de pinheira (Annona squamosa L.). Rev Ceres. 2006 Jul/Ago;53(308):439-445.

11. Empresa Brasileira de Pesquisa Agropecuária. Serviço Nacional de Levantamento e Conservação do solo. Manual de métodos de análise de solos. Rio de Janeiro: Embrapa Solos, 2011, 230p.

12. Maguire JD. Speed of germination: aid in selection and evaluation for seedling emergence and vigour. Crop Science. 1962;2(2):176-177.

13. Ferreira DF. Sisvar: a Guide for its Bootstrap procedures in multiple comparisons. Ciênc Agrotec. 2014 Mar/Abr;38(2):109-112, doi: 10.1590/S1413-70542014000200001.

14. Sacramento CK, Moura JIL, Coelho Junior E. Cultivo da Graviola. In: Santos-Serejo JA, Dantas JLL, Coelho CVS, Coelho YS (Org.). Fruticultura tropical: espécies regionais e exóticas. Brasília: Embrapa Informação Tecnológica, 2009, p. 95-132.

15. Oliveira IVM, Andrade RA, Martins ABG. Influência da temperatura na germinação de sementes de Annona montana. Rev Bras Frutic. 2005 Ago;27(2):344-345, doi: 10.1590/S0100-29452005000200041

16. Andrade ACS, Souza AF, Ramos FN, Pereira TS, Cruz APM. Germinação de sementes de jenipapo: temperatura, substrato e morfologia do desenvolvimento pós-seminal. Pesq Agropec Bras. 2000 Mar;35(3):609-615, doi: 10.1590/S0100-204X2000000300017.

17. Bewley JD, Bradford KJ, Hilhorst HWM, Nonogaki H. Seeds: physiology of development, germination and dormancy. 3 ed. New York: Springer, 2013. 392p.

18. Braga Júnior JM, Bruno RLA, Alves EU. Emergência de plântulas de Zizyphus joazeiro Mart (Rhamnaceae) em função de substratos. Rev Árvore. 2010;34(4):609-616, doi: 10.1590/S010067622010000400005

19. Guimarães IP, Coelho MFB, Benedito CP, Maia SSS, Nogueira CSR, BATISTA PF. Efeito de diferentes substratos na emergência e vigor de plântulas de mulungu. Bioscience J. 2011 Nov/Dec;27(6):932-938.

20. Oliveira NVM, Cavalcante IHL, Martins ABG. Influência do substrato na emergência de plântulas de sapota preta. Rev Caatinga. 2006 Out/Dec;19(4):383-386. 